\title{
ON THE KLEINIAN CONSTRUCTION OF ABELIAN FUNCTIONS OF CANONICAL ALGEBRAIC CURVES
}

\author{
J C EILBECK, V Z ENOLSKII, AND D V LEYKIN
}

\section{INTRODUCTION}

The discovery of classical and quantum completely integrable systems led to an increase in interest in the theory of Abelian functions in theoretical physics and applied mathematics. This area was considered traditionally as a field of pure mathematics. This new trend makes it necessary to reconsider classical results in the area from the point of view of modern applications.

In this paper we consider an arbitrary algebraic curve $V$ of genus $g$ and construct the field of meromorphic functions on its Jacobi variety $\operatorname{Jac}(V)$ in terms of Kleinian $\wp$-functions,

$$
\wp_{i j}(u)=-\frac{\partial^{2}}{\partial u_{i} \partial u_{j}} \ln \sigma(u), \quad i, j=1, \ldots, g,
$$

where the vector $u \in \operatorname{Jac}(V)$ and $\sigma$ is the Kleinian $\sigma$-function. The effective construction of the $\sigma$-function plays the principal role in our approach. It is defined on the universal space of Jacobians, which is the fibration with the base given by the space of moduli, $M(V)$ of the curve $V$ of dimension $d \leq 3 g-3$ and a fibre generated by Jacobi variety $\operatorname{Jac}(V)$. The Kleinian $\sigma$ function represents a natural generalization of the Weierstrass elliptic function to the case of an arbitrary algebraic curve, and has the following properties:

- The $\sigma$-function is automorphic with respect to the action of the symplectic group $\operatorname{Sp}(2 g, \mathbb{Z})$.

- The $\sigma$-function is an entire function on the universal space and is expanded in a power series whose entries are monomials

$$
u_{1}^{\alpha_{1}} \cdots u_{g}^{\alpha_{g}} \lambda_{1}^{\beta_{1}} \cdots \lambda_{d}^{\beta_{d}}
$$

where $u \in \operatorname{Jac}(V), \lambda \in M(V), \alpha \in\left(\mathbb{Z}_{+}\right)^{g}, \beta \in\left(\mathbb{Z}_{+}\right)^{d},\left(\mathbb{Z}_{+}\right)=0 \cup \mathbb{N}$.

The research described in this publication was supported in part by grants from the Civil Research Development Foundation, CRDF grant no. UM1-325, the INTAS grant no. 96770 and the Engineering and Physical Sciences Research Committee, grant GR/L06119 (JCE and VZE). 
- The addition formula for the $\sigma$ function inherits the form of the addition theorem for the Weierstrass $\sigma$ function and is written as

$$
\frac{\sigma(u+v) \sigma(u-v)}{\sigma(u)^{2} \sigma(v)^{2}}=\text { polynomial in } \wp_{i, j}
$$

This construction began with Weierstrass [22, 23] and Klein [13] and was well documented in $[1,2]$; recently B.M.Buchstaber and two of the authors reviewed the hyperelliptic case[4, 5, 6].

The principal result of $[4,5]$ is the discovery of a $(g+2) \times(g+2)$ matrix $H$ of a rank $\leq 4$ whose entries are $\wp$ functions and the moduli of the curve $V$ and which dictates all the theory: the $4 \times 4$ minors give the embedding of the Kummer variety $\operatorname{Kum}(V)$ in the projective space, $3 \times 3$ minors describe in the same way the Jacobi variety $\operatorname{Jac}(V)$, and the associated $\mathrm{KdV}$ hierarchy is constructed in terms of $2 \times 2$ minors of the matrix $H$. The hyperelliptic Kleinian functions were also developed in $[16,10]$ for a description of the lattice KdV system.

The present paper is aimed at developing the analogous matrix realization of the Kleinian construction of Abelian functions for an arbitrary algebraic curve, including the case of a singular curve. The paper is based on the recent results of [7], where the construction of the Kleinian $\sigma$ functions was given for a non-hyperelliptic curve, and contains a set of explicit formulae to realize the approach of [7].

The paper starts from an example of a hyperelliptic curve of genus two for which we give the basic formulae of the theory and their application to completely integrable systems. We concentrate further on the construction of the principle objects - Kleinian $\sigma$ functions for a wide class of algebraic curves (the so called canonical curves). With this purpose we derive the canonical abelian differentials using the Weierstrass gap theorem as the main working tool. We obtain as the result the Kleinian formula (3.28), which is a generating one for deriving the complete set relations between $\wp$ functions and their derivatives. The principle result of the paper is the explicit solution of the Jacobi inversion problem, which is an alternative to that given by M.Nöther [17]. We consider as a main example the case of a non singular trigonal curve, for which we give the complete set of formulae, analogous to those given in $[1,2]$ for hyperelliptic curve. The paper is completed by a short discussion on the application of our approach to completely integrable equations and of the further perspectives of the development of the theory. 
2. KLEINIAN FUnCTIONS OF THE GENUS TWO HYPERELLIPTIC CURVE

In this section we consider the simplest example - the Kleinian functions of an algebraic curve of genus two, and demonstrate how these functions work in completely integrable systems.

We consider the Riemann surface of a curve $V(x, y)$ of genus $g=2$,

$$
\begin{aligned}
y^{2} & =4 x^{5}+\lambda_{4} x^{4}+\lambda_{3} x^{2}+\lambda_{2} x^{2}+\lambda_{1} x+\lambda_{0} \\
& =4 \prod_{k=1}^{5}\left(x-a_{k}\right), \quad a_{i} \neq a_{j} \in \mathbb{C}
\end{aligned}
$$

equipped with a homology basis $\left(\mathfrak{a}_{1}, \mathfrak{a}_{2} ; \mathfrak{b}_{1}, \mathfrak{b}_{2}\right) \in H_{1}(V, \mathbb{Z})$.

Introduce the canonical basis in the space of holomorphic differentials $\mathrm{d} \mathbf{u}^{T}=\left(\mathrm{d} u_{1}, \mathrm{~d} u_{2}\right) \in \mathcal{H}^{1}(V, \mathbb{C})$ as follows

$$
\mathrm{d} u_{1}=\frac{\mathrm{d} x}{y}, \quad \mathrm{~d} u_{2}=\frac{x \mathrm{~d} x}{y} .
$$

The associated canonical meromorphic differentials of the second kind $\mathrm{d} r^{T}=$ $\left(\mathrm{d} r_{1}, \mathrm{~d} r_{2}\right)$ have the form

$$
\mathrm{d} r_{1}=\frac{\lambda_{3} x+2 \lambda_{4} x^{2}+12 x^{3}}{4 y} d x, \quad \mathrm{~d} r_{2}=\frac{x^{2}}{y} d x
$$

The $2 \times 2$ matrices of their periods,

$$
\begin{aligned}
2 \omega & =\left(\oint_{\mathfrak{a}_{k}} \mathrm{~d} u_{l}\right)_{k, l=1,2}, \quad 2 \omega^{\prime}=\left(\oint_{\mathfrak{b}_{k}} \mathrm{~d} u_{l}\right)_{k, l=1,2}, \\
2 \eta & =\left(\oint_{\mathfrak{a}_{k}} \mathrm{~d} r_{l}\right)_{k, l=1,2}, \quad 2 \eta^{\prime}=\left(\oint_{\mathfrak{b}_{k}} \mathrm{~d} r_{l}\right)_{k, l=1,2}
\end{aligned}
$$

satisfy the equations,

$$
\omega^{\prime} \omega^{T}-\omega \omega^{\prime T}=0, \quad \eta^{\prime} \omega^{T}-\eta \omega^{\prime T}=-\frac{l \pi}{2} 1_{2}, \quad \eta^{\prime} \eta^{T}-\eta \eta^{\prime T}=0,
$$

which generalizes the Legendre relations between complete elliptic integrals to the case $g=2$.

The fundamental $\sigma$ function in this case is a natural generalization of the Weierstrass elliptic $\sigma$ function and is defined as follows

$$
\begin{aligned}
\sigma(u) & =\frac{\pi}{\sqrt{\operatorname{det}(2 \omega)}} \frac{\varepsilon}{\sqrt[4]{\prod_{1 \leq i<j \leq 5}\left(a_{i}-a_{j}\right)}} \\
& \times \exp \left\{u^{T} \eta(2 \omega)^{-1} u\right\} \theta[\varepsilon]\left((2 \omega)^{-1} u \mid \omega^{\prime} \omega^{-1}\right),
\end{aligned}
$$


where $\varepsilon^{8}=1$ and $\theta[\varepsilon](v \mid \tau)$ is the $\theta$ function with an odd characteristic $[\varepsilon]=$ $\left[\begin{array}{ll}\varepsilon_{1} & \varepsilon_{2} \\ \varepsilon_{1}^{\prime} & \varepsilon_{2}^{\prime}\end{array}\right]$

$$
\theta[\varepsilon](v \mid \tau)=\sum_{m \in \mathbb{Z}^{2}} \exp \imath \pi\left\{(m+\varepsilon)^{T} \tau(m+\varepsilon)+2\left(v+\varepsilon^{\prime}\right)^{T} \tau(m+\varepsilon)\right\}
$$

We denote

$$
\begin{gathered}
\wp_{11}(u)=-\frac{\partial^{2}}{\partial u_{1}^{2}} \ln \sigma(u), \quad \wp_{12}(u)=-\frac{\partial^{2}}{\partial u_{1} \partial u_{2}} \ln \sigma(u), \\
\wp_{22}(u)=-\frac{\partial^{2}}{\partial u_{2}^{2}} \ln \sigma(u) .
\end{gathered}
$$

The multi-index symbols $\wp_{i, j, k}$ etc. are defined as logarithmic derivatives by the variable $u_{i}, u_{j}, u_{k}$ on the corresponding indices $i, j, k$ etc.

The equations of the Jacobi inversion problem,

$$
\begin{aligned}
& u_{1}=\int_{a_{1}}^{x_{1}} \mathrm{~d} u_{1}+\int_{a_{2}}^{x_{2}} \mathrm{~d} u_{1}, \\
& u_{2}=\int_{a_{1}}^{x_{1}} \mathrm{~d} u_{2}+\int_{a_{2}}^{x_{2}} \mathrm{~d} u_{2},
\end{aligned}
$$

are equivalent to an algebraic equation

$$
\mathcal{P}(x, u)=x^{2}-\wp_{22}(u) x-\wp_{12}(u)=0,
$$

that is, the pair $\left(x_{1}, x_{2}\right)$ is the pair of roots of (2.3). So we have

$$
\wp_{22}(u)=x_{1}+x_{2}, \wp_{12}(u)=-x_{1} x_{2} .
$$

The corresponding $y_{i}$ is expressed as

$$
y_{i}=\wp_{222}(u) x_{i}+\wp_{122}(u), \quad i=1,2 .
$$

There is the following expression for the function $\wp_{11}(u)$ in terms of $x_{1}, x_{2}$ and $y_{1}, y_{2}$ :

$$
\wp_{11}(u)=\frac{F\left(x_{1}, x_{2}\right)-2 y_{1} y_{2}}{4\left(x_{1}-x_{2}\right)^{2}}
$$

where

$$
F\left(x_{1}, x_{2}\right)=\sum_{r=0}^{2} x_{1}^{r} x_{2}^{r}\left[2 \lambda_{2 r}+\lambda_{2 r+1}\left(x_{1}+x_{2}\right)\right] .
$$

All the possible pairwise products of the $\wp_{i j k}$ functions are expressed as follows in terms of $\wp_{22}, \wp_{12}, \wp_{11}$ and constants $\lambda_{s}$ of the defining equation 
(2.1). We give here only basis equations

$$
\begin{aligned}
\wp_{222}^{2} & =4 \wp_{11}+\lambda_{3} \wp_{22}+4 \wp_{22}^{3}+4 \wp_{12} \wp_{22}+\lambda_{4} \wp_{22}^{2}+\lambda_{2} \\
\wp_{222} \wp_{122} & =\frac{1}{2} \lambda_{1}+2 \wp_{12}^{2}-2 \wp_{11} \wp_{22}+\frac{1}{2} \lambda_{3} \wp_{12} \\
& +4 \wp_{12} \wp_{22}^{2}+\lambda_{4} \wp_{12} \wp_{22}, \\
\wp_{122}^{2} & =\lambda_{0}-4 \wp_{11} \wp_{12}+\lambda_{4} \wp_{12}^{2}+4 \wp_{22} \wp_{12}^{2} .
\end{aligned}
$$

All such expressions may be rewritten in the form of an extended cubic relation as follows. For arbitrary $l, k \in \mathbb{C}^{4}$ the following formula is valid [2]

$$
l^{T} \pi \pi^{T} k=-\frac{1}{4} \operatorname{det}\left(\begin{array}{cc}
H & l \\
k^{T} & 0
\end{array}\right),
$$

where $\pi^{T}=\left(\wp_{222},-\wp_{221}, \wp_{211},-\wp_{111}\right)$ and $H$ is the $4 \times 4$ matrix:

$$
H=\left(\begin{array}{cccc}
\lambda_{0} & \frac{1}{2} \lambda_{1} & -2 \wp_{11} & -2 \wp_{12} \\
\frac{1}{2} \lambda_{1} & \lambda_{2}+4 \wp_{11} & \frac{1}{2} \lambda_{3}+2 \wp_{12} & -2 \wp_{22} \\
-2 \wp_{11} & \frac{1}{2} \lambda_{3}+2 \wp_{12} & \lambda_{4}+4 \wp_{22} & 2 \\
-2 \wp_{12} & -2 \wp_{22} & 2 & 0
\end{array}\right) .
$$

The vector $\pi$ satisfies the equation $H \pi=0$, and so the functions $\wp_{22}, \wp_{12}$ and $\wp_{11}$ are related by the equation

$$
\operatorname{det} H=0 .
$$

The equation (2.9) defines the quartic Kummer surface $\mathbb{K}$ in $\mathbb{C}^{3}$ [12].

The $\wp_{i j k l}$ functions are expressed as follows

$$
\begin{aligned}
& \wp_{2222}=6 \wp_{22}^{2}+\frac{1}{2} \lambda_{3}+\lambda_{4} \wp_{22}+4 \wp_{12}, \\
& \wp_{2221}=6 \wp_{22} \wp_{12}+\lambda_{4} \wp_{12}-2 \wp_{11}, \\
& \wp_{2111}=6 \wp_{22} \wp_{11}+4 \wp_{12}^{2}+\frac{1}{2} \lambda_{3} \wp_{12} . \\
& \wp_{1111}=6 \wp_{11}^{2}-3 \lambda_{0} \wp_{22}+\lambda_{2} \wp_{12}-\frac{1}{2} \lambda_{1} \wp_{12}+\lambda_{0},
\end{aligned}
$$

These equations can be identified with completely integrable partial differential equations and dynamical systems, which are solved in terms of Abelian functions of hyperelliptic curve of genus two.

To demonstrate this statement we consider the stationary Veselov-Novikov equation,

$$
\begin{aligned}
& \alpha u_{x x x}+\beta u_{y y y}-3 \alpha(v u)_{x}-3 \beta(w u)_{y}=0, \\
& w_{x}=u_{y}, \quad v_{y}=u_{x}
\end{aligned}
$$


where $\alpha, \beta \neq 0$ are constants. Then the following proposition is valid

Proposition 2.1. The stationary flow of the Veselov-Novikov equation is satisfied if we set

$$
\begin{aligned}
u(x, y) & =2 \wp_{22}(x, y), \\
v(x, y) & =2 \wp_{12}(x, y)+\frac{1}{3}\left(\lambda_{4}-\frac{\beta}{\alpha}\right), \\
w(x, y) & =2 \wp_{11}(x, y)+\frac{1}{3}\left(\lambda_{2}-\frac{\alpha}{\beta}\right) .
\end{aligned}
$$

Proof. A straightforward substitution of (2.16) into (2.15) and use of the relations (2.11), (2.13) and $H \pi=0$.

Because the Kleinian functions $\wp_{22}, \wp_{12}, \wp_{11}$ are the coordinates of the Kummer surface, the stationary Veselov-Novikov equation, being associated with a hyperelliptic curve of genus two, describes the Kummer surface. The link between the stationary Veselov-Novikov equation and the Kummer surface was recently discussed by Ferapontov [11].

It can be also shown, that the equations (2.10)-(2.14) describe hierarchies of KdV and "sine-Gordon" equations associated with the hyperelliptic curve of genus two.

In what follows we develop the Kleinian construction of Abelian functions for a certain class of non-hyperelliptic algebraic curves.

\section{Kleinian CONSTRUCtion of AbELIAN FUnCtions}

3.1. The curve. Let $V$ be an algebraic curve given by an irreducible equation

$$
f(x, y)=0, \quad f(x, y)=a_{0}(x) y^{n}-\sum_{k=1}^{n} a_{k}(x) y^{n-k},
$$

where $a_{k}(x)$ are polynomials in $x$ and $a_{0}(x)$ and $a_{k}(x), k=1 \ldots, n$ have no common factors. The curve is called singular if $a_{0}(x) \not \equiv 1$ and nonsingular otherwise. In other words, a singular curve is the curve which has points $(y=\infty, x \neq \infty)$.

Definition 3.1. The order $N$ of an arbitrary rational function $\phi(x, y)$ on the curve $V$ is the number $N$ of common solutions $\left(x_{1}, y_{1}\right), \ldots,\left(x_{N}, y_{N}\right)$ of the equations

$$
f(x, y)=0, \quad \phi(x, y)=0 .
$$

We shall call the positive integers $(n, s)$ orders of the curve $V$ because, clearly $x$ is a function of order $n$ and $y$ is a function of order $s$.

The definitions given above permit us to formulate the Weierstrass gap theorem, which serves as a principal working tool in what follows. 
Theorem 3.1 (Weierstrass Lückensatz). For $V$ there exists a number $g$ of positive integers $n_{1}, \ldots n_{g}$ ("gap sequence") such that for each number $n_{i}$ the equations

$$
f(x, y)=0, \quad \psi(x, y)=0, \quad \operatorname{ord}_{V} \psi(x, y)=n_{i}
$$

have no solutions and

- all exceptional integers ("gaps") belong to the interval $[1,2 g-1]$

$$
0<n_{1}<n_{2}<\ldots<n_{g}<2 g .
$$

- if the numbers $n_{i}$ and $n_{j}$ - are permitted ("non-gaps") then the numbers $p n_{i}+q n_{j}$, where $p, q \in \mathbb{N}$ are also non-gaps.

The following corollaries are valid

Corollary 3.1.1. Consider the gap sequence with the number of gaps $g$. Let $n$ be the lowest from the non-gaps. Denote by $s_{i}, i=1, \ldots n-1$ the lowest non-gaps such that $s_{i}(\bmod n)=i<n$. Then the following equality is valid

$$
\sum_{i=1}^{n-1}\left[\frac{s_{i}}{n}\right]=g \text {. }
$$

Corollary 3.1.2. Suppose that the condition of Corollary 3.1.1 are satisfied. Let $s=\min \left\{s_{1}, \ldots, s_{n-1}\right\}$. Then the following equality is valid

$$
\frac{(n-1)(s-1)}{2}-g=\delta, \quad \text { where } \delta \geq 0
$$

The Weierstrass gap theorem introduced two important positive integers $g$ and $\delta$ which are the principal geometrical invariants of the curve.

Definition 3.2. The nonnegative integer $\delta$ is the number of multiple points of the curve, which can not exceed $(n-1)(s-1) / 2$. The number of gaps $g$, which is the difference between the maximal number of multiple points of a curve of orders $(n, s)$ and number $\delta$ of multiple points, which it actually has, is called the genus ${ }^{1}$ of the curve.

To describe the number $\delta$ analytically we compute the discriminant (in $y$ ) $D_{y}(x)$ of the curve (3.1),

$$
D_{y}(x)=\prod_{i=1}^{n-1} d_{i}^{\varepsilon_{i}}(x) r(x),
$$

where $\varepsilon \in \mathbb{N} \geq 2$. Zeros of $d_{i}(x) i=1, \ldots, n-1$, where

$$
d_{i}(x)=\prod_{j=1}^{\delta_{j}}\left(x-\kappa_{i j}\right), \quad \delta_{j} \in \mathbb{N}, \quad \kappa_{i j} \in \mathbb{C}
$$

\footnotetext{
${ }^{1}$ In classical literature - Geschlecht or deficiency
} 
are multiple points, whence zeros of the polynomial $r(x)$ are the branching points of the curve. Let us write the number

$$
\delta=\sum_{i=1}^{n-1} \delta_{i}\left(\varepsilon_{i}-1\right) \in \mathbb{Z}_{+},
$$

where we denote $\mathbb{Z}_{+}=\mathbb{N} \oplus 0$, as number of multiple points. The curve is called non-degenerate if $\delta=0$ and degenerate otherwise.

Introduce on $V$ the structure of one-dimensional compact complex manifold - the Riemann surface of algebraic curve by introducing a local parametrization of the point $(y, x)=(y(\xi), x(\xi))$ in the vicinity of a point $(a, b)$, where $\xi$ is the local coordinate :

$$
(y, x)= \begin{cases}(a+\xi, b+\xi) & \text { if }(a, b) \text { is an regular point } \\ \left(a+\xi, b+\xi^{m}\right) & \text { if }(a, b) \text { is an branching point } \\ \left(\frac{1}{\xi^{s}}, \frac{1}{\xi^{n}}\right) & \text { if }(a=\infty, b=\infty) \text { is branching } \\ \left(a+\xi^{m}, b+\xi\right) & \text { if }(a, b) \text { is an multiple point } \\ & \text { of multiplicity } m .\end{cases}
$$

We will employ further the same notation for the plane curve and the Riemann surface $-V$. Combining notions introduced so far, we come up with

Definition 3.3. The algebraic curve $V$ of orders $(n, s)$ is called nonsingular non-degenerate canonical iff $a_{0}(x)=1, \delta=0$ and $(n, s)$ are co-prime.

It is clearly that the Weierstrass gap sequence of the nonsingular nondegenerate canonical curve $V$ of orders $(n, s)$ is generated by two coprime integers $n$ and $s$. Alternatively, the Weierstrass gap sequence of a nonsingular degenerate canonical curve $V$ of orders $(n, s)$ is generated by the integers $n$ and $s_{1}=s, \ldots, s_{k}$, where $1<k \leq n-1$.

The canonical algebraic curve has a branching point at infinity, where the coordinates $x$ and $y$ are given as $x=\frac{1}{\xi^{n}}=\infty, \quad y=\frac{1}{\xi^{s}}=\infty, \quad 2<$ $n<s$, where $\xi$ is the local coordinate. The polynomial $f(x, y)$ defining the canonical curve can be written in the form

$$
f(x, y)=y^{n}-x^{s}+\text { terms of lower order. }
$$

Let us fix in the form of a proposition the important property of the nonsingular curve

Proposition 3.2. The order of a monomial $x^{P} y^{Q}, P, Q \in \mathbb{N}$ on nonsingular non-degenerate curve of orders $(n, s)$ equals

$$
\operatorname{ord}_{V} x^{P} y^{Q}=n P+s Q
$$


Example 3.1. Consider all canonical curves of genus $g=4$ and construct the associated Weierstrass gap sequences.

- Nonsingular canonical hyperelliptic curve

$$
\overline{0}, 1, \overline{2}, 3, \overline{4}, 5, \overline{6}, 7, \overline{8,9, \cdots}
$$

The sequence is generated by the orders 2 and 9 .

- Nonsingular canonical trigonal curve

$$
\overline{0}, 1,2, \overline{3}, 4, \overline{5,6}, 7, \overline{8,9, \cdots}
$$

The sequence is generated by the orders 3 and 5 .

- Singular canonical trigonal curve

$$
\overline{0}, 1,2, \overline{3}, 4,5, \overline{6,7,8, \cdots}
$$

The sequence is generated by the orders 3,7 and 8 .

- Singular canonical four-sheeted covering

$$
\overline{0}, 1,2,3, \overline{4,5,6}, 7, \overline{8,9, \cdots}
$$

The sequence is generated by the orders 4,5 and 6 .

3.2. The differentials and integrals. All the construction is based on the explicit realization of the fundamental second order 2-differential.

Definition 3.4. The 2-differential $\mathrm{d} \Omega(y, x ; w, z)$ on $V \times V$ is called a fundamental second order 2-differential if it is symmetric $\mathrm{d} \Omega(x, y ; z, w)=$ $\mathrm{d} \Omega(z, w ; x, y)$ and has the only pole of the second order along the diagonal, $x=z$ in the vicinity of which it can be given as

$$
\mathrm{d} \Omega(y, x ; w, z)=\frac{\mathrm{d} \xi \mathrm{d} \eta}{(\xi-\eta)^{2}}+O(1) \mathrm{d} \xi \mathrm{d} \eta,
$$

where $\xi, \eta-$ are local coordinates of the points $x$ and $z$ respectively. We shall look for a realization of $\mathrm{d} \Omega(y, x ; w, z)$ in the form [1]

$$
\mathrm{d} \Omega(y, x ; w, z)=\frac{F(y, x ; w, z) \mathrm{d} x \mathrm{~d} z}{(x-z)^{2} f_{y}(x, y) f_{w}(z, w)},
$$

where $F(y, x ; w, z)$ is a polynomial of its variables.

Holomorphic differentials can be represented locally at every point $(X, Y) \in V$ in the form $\mathrm{d} u=h(\xi) \mathrm{d} \xi$, where $h-$ is the holomorphic function and $\xi-$ is the local parameter in the vicinity of the point $(X, Y)$. For the algebraic curve of genus $g$ there exist exactly $g$ independent holomorphic differentials, which can be written in the form

$$
\mathrm{d} u_{i}=\frac{x^{P_{i}} y^{Q_{i}} \mathrm{~d} x}{f_{y}(x, y)}, \quad i=1 \ldots, g,
$$


where $\operatorname{ord}_{V} x^{P_{i}} y^{Q_{i}}, i=1, \ldots, g$ are exactly $g$ first non-gaps of the Weierstrass gap sequence. Introduce the $g$-vector

$$
\mathfrak{U}^{T}=\left(\ldots, \mathcal{U}_{i, j}, \ldots\right)=\left(\mathcal{U}_{1}, \ldots, \mathcal{U}_{g}\right),
$$

whose components $\mathcal{U}_{i, j}=x^{P_{i}}{ }^{Q_{i}}$ are ordered by increasing order of the monomials:

$$
\mathcal{U}_{1}=\mathcal{U}_{0,0}=1, \mathcal{U}_{2}=\mathcal{U}_{1,0}=x, \ldots
$$

Definition 3.5. Let $\mathcal{P}(x)$ be a polynomial in $x$ of order $\operatorname{ord}_{x} \mathcal{P}(x)=n$ and $F_{k}(x)$ polynomials of orders $k$, such that the equality

$$
\frac{\mathcal{P}(x)-\mathcal{P}(z)}{x-z}=\sum_{k=0}^{n} z^{n-k} F_{k}(x)
$$

is valid. Then $D_{z}^{k} \mathcal{P}(z)=F_{k}(x)$ is the umbral derivative of order $k$ of the polynomial $\mathcal{P}(z)$.

Definition 3.6. The rational function $\Psi(x, y)$ on the nonsingular curve $V$ is called an entire rational function, if $\Psi(x, y) \rightarrow \infty$ iff $x \rightarrow \infty$. Entire rational functions generate a ring, which we denote by $\mathcal{O}(V)$.

Let

$$
\psi(x, y)^{T}=\left(1, \psi_{1}(x, y), \ldots, \psi_{n-1}(x, y)\right)
$$

be a basis in $\mathcal{O}(V)$, where

$$
\psi_{i}(x, y)=\left(\frac{D_{y}^{n-i} f(x, y)}{d_{i}(x)}\right), \quad i=0, \ldots, n-1,
$$

where $D_{y}^{k}$ - is the umbral derivative in $y$ of order $k$, and $f(x, y)$ is the polynomial defining the curve.

Then it follows from the definition of the umbral derivative and the existence of such functions

$$
\phi(x, y)^{T}=\left(\phi_{0}(x, y), \phi_{1}(x, y), \ldots, \phi_{n-1}(x, y)\right),
$$

that

$$
\psi(x, y)^{T} \phi(x, Y)=\frac{f(x, Y)-f(x, y)}{Y-y} . .
$$

The functions $\phi_{i}$ have, clearly, the form

$$
\phi_{i}(x, y)=d_{i}(x) y^{n-i-1}, \quad i=0, \ldots, n-1 .
$$


The property (3.15) allows us to construct $A$ canonical differential of the third kind with unique poles of the first order in the points $\left(x_{1}, y_{1}\right)$ and $\left(x_{2}, y_{2}\right)$ as follows [1]

$$
\mathrm{d} \Pi_{x_{1}, x_{2}}(x)=\frac{\mathrm{d} x}{f_{y}(x, y)}\left\{\frac{\psi\left(x_{1}, y_{1}\right)^{T} \phi(x, y)}{x-x_{1}}-\frac{\psi\left(x_{2}, y_{2}\right)^{T} \phi(x, y)}{x-x_{2}}\right\} .
$$

To construct explicitly the fundamental second order 2-differential $\mathrm{d} \Omega(x, y ; z, w)$ we consider on $V \times V$ the 2-differential

$$
\mathrm{d} z \frac{\mathrm{d} x}{f_{y}(x, y)} \frac{\partial}{\partial z} \frac{\psi^{T}(z, w) \phi(x, y)}{x-z} .
$$

It follows from the definition of the umbral derivatives, that this differential has a pole of the second order along the diagonal, where it is represented in the form (3.9). But being holomorphic in $(x, y)$ away from the diagonal, this differential has nevertheless poles in the variables $(z, w)$ at $z=\infty$. Restore the symmetry by setting

$$
\begin{aligned}
\mathrm{d} \Omega(x, y ; z, w) & =\mathrm{d} z \frac{\mathrm{d} x}{f_{y}(x, y)} \frac{\partial}{\partial z} \frac{\psi^{T}(z, w) \phi(x, y)}{x-z} \\
& +\mathcal{R}^{T}(z, w) \mathcal{U}(x, y) \frac{\mathrm{d} x \mathrm{~d} z}{f_{y}(x, y) f_{w}(z, w)},
\end{aligned}
$$

where the $g$-vector $\mathfrak{U}(x, y)$ is defined by the holomorphic differentials and the $g$-vector $\mathcal{R}^{T}=\left(\mathcal{R}_{1}, \ldots, \mathcal{R}_{g}\right)$ is found from the equality

$$
\begin{aligned}
& \frac{\partial f(z, w)}{\partial w} \frac{\partial}{\partial z} \frac{\psi^{T}(z, w) \phi(x, y)}{x-z}-\frac{\partial f(x, y)}{\partial y} \frac{\partial}{\partial x} \frac{\psi^{T}(x, y) \phi(z, w)}{z-x} \\
& =\mathcal{R}^{T}(x, y) \mathfrak{U}(z, w)-\mathfrak{U}^{T}(x, y) \mathcal{R}(z, w)
\end{aligned}
$$

The canonical differentials of the second kind $\mathrm{d} \mathbf{r}^{T}=\left(\mathrm{d} r_{1}, \ldots, \mathrm{d} r_{g}\right)$ associated with the holomorphic differentials (3.11) are then given by the formula

$$
\mathrm{d} r_{i}=\frac{\mathcal{R}_{i}(x, y) \mathrm{d} x}{f_{y}(x, y)}, \quad i=1 \ldots, g
$$

where the correspondence

$$
\mathcal{R}_{i}(x, y) \leftrightarrow \mathcal{U}_{i}(x, y), \quad i=1, \ldots, g
$$

of the polynomials $\mathcal{R}(x, y)_{i}$ with monomials $\mathcal{U}_{i}(x, y)$ being ordered according to the Weierstrass gap sequence as was pointed in (3.12) is supposed.

The Weierstrass gap sequence gives complete information about the curve. Consider the Weierstrass gap sequence and prescribe by the lowest non-gap entries $(n, s), n<s \operatorname{orders~ord}_{V} x=n$ and $\operatorname{ord}_{V} y=s$. Write the curve in the form (3.1). 
Define the functions (3.13). $d_{0}=1$, and $d_{1}(x), \ldots, d_{n-1}(x)$ polynomials in $x$, such that $\operatorname{ord}_{V} \psi_{i}(x, y)=s_{i}, i=1, \ldots, n-1$ are the lowest non-gaps, and that $s_{i}(\bmod n)<i<n$. According to Theorem, the functions

$$
\psi_{0}(x, y)=1, \psi_{1}(x, y), \ldots, \psi_{n-1}(x, y)
$$

give a basis in $\mathcal{O}(V)$. Therefore for every pair $i, k=0, \ldots, n-1$ the following equalities are valid

$$
\psi_{i}(x, y) \psi_{k}(x, y)=\sum_{j=0}^{n-1} \alpha_{i k}^{j}(x) \psi_{j}(x, y),
$$

where $\alpha_{i k}^{(j)}(x)$ are polynomials in $x$. By equating powers of $y$ in equations (3.20) one can find the form of the polynomials $A_{k}$ entering the equation of the curve (3.1) and also the property, which the polynomials $d_{i}(x)$ possess

$$
d_{i-1}(x) d_{i+1}(x) \text { divisible by } d_{i}^{2}(x)
$$

As result we come to the following result:

Proposition 3.3. The curve $V$ of orders $(n, s)$ is given by the formula

$$
a_{0}(x) y^{n}-\sum_{k=0}^{n-1} a_{k}(x) \prod_{i=2}^{n-1} \tilde{d}_{i}^{n-k}(x) y^{k-1}=0,
$$

where $a_{k}(x)$ are polynomials of orders ord $_{x} a_{k}(x) \leq s$, and the polynomials $\tilde{d}_{i}(x)$ are defined by the equation

$$
\tilde{d}_{i+1}=\frac{d_{i-1}(x) d_{i+1}(x)}{d_{i}^{2}(x)}, \quad i=1 . . n-1 .
$$

where the polynomials $d_{i}$ posses the property (3.21).

This construction can be inverted: the Weierstrass gap sequence is uniquely defined by the curve and can be algorithmically constructed by the curve.

3.3. Kleinian Formula. Let $(\mathfrak{a}, \mathfrak{b})$ be a basis of cycles in $\mathrm{H}_{1}(V, \mathbb{Z})$ with the intersection matrix $\mathfrak{a}_{i} \circ \mathfrak{a}_{k}=0$ and $\mathfrak{b}_{i} \circ \mathfrak{b}_{k}=0 \quad \mathfrak{a}_{i} \circ \mathfrak{b}_{k}=-\mathfrak{b}_{k} \circ \mathfrak{a}_{i}=1$. $g \times g$ matrices of their periods $\mathfrak{a} \mathfrak{b}$-periods,

$$
2 \omega=\left(\oint_{\mathfrak{a}_{k}} \mathrm{~d} u_{l}\right)_{k, l=1, \ldots, g}, \quad 2 \omega^{\prime}=\left(\oint_{\mathfrak{b}_{k}} \mathrm{~d} u_{l}\right)_{k, l=1, \ldots, g}
$$

are non-degenerate and the matrix $\tau=\omega^{\prime} \omega^{-1}$ is symmetric and has positive imaginary part. Under the action of the transformation $(2 \omega)^{-1}$ the vector $\mathrm{d} \mathbf{u}=\left(\mathrm{d} u_{1}, \ldots, \mathrm{d} u_{g}\right)^{T}$ becomes the vector of normalised holomorphic differentials $\mathrm{d} \mathbf{v}=\left(\mathrm{d} v_{1}, \ldots, \mathrm{d} v_{g}\right)^{T}$, namely the vector $H^{1}(V, \mathbb{C})$ satisfying the 
conditions $\oint_{\mathfrak{a}_{k}} \mathrm{~d} v_{k}=\delta_{k l}, k, l=1 \ldots, g$. Introduce also the period matrices of the canonical differentials of the second kind

$$
\left(2 \eta, 2 \eta^{\prime}\right)=\left(\left\{-\oint_{\mathfrak{a}_{i}} \mathrm{~d} r_{j}\right\},\left\{-\oint_{\mathfrak{b}_{i}} \mathrm{~d} r_{j}\right\}\right)_{i, j=1, \ldots, n} .
$$

Denote by $\operatorname{Jac}(V)$ the Jacobian of the curve $V$, which is the factor $\mathbb{C}^{g} / \Gamma$, where $\Gamma=2 \omega \oplus 2 \omega^{\prime}$ is the lattice, generated by the periods of the holomorphic differentials.

Divisors on Riemann surfaces are given by formal sums of analytic points $\mathcal{D}=\sum_{i}^{n} m_{i}\left(y_{i}, x_{i}\right)$, and $\operatorname{deg} \mathcal{D}=\sum_{i}^{n} m_{i}$. The effective divisor is such that $m_{i}>0 \forall i$. Let $\mathcal{D}$ be a divisor of degree $0, \mathcal{D}=\mathcal{X}-\mathcal{Z}$, with $\mathcal{X}$ and $\mathcal{Z}$ the effective divisors $\operatorname{deg} X=\operatorname{deg} Z=n$ given by $X=\left\{\left(y_{1}, x_{1}\right), \ldots,\left(y_{n}, x_{n}\right)\right\}$ and $Z=\left\{\left(w_{1}, z_{1}\right), \ldots,\left(w_{n}, z_{n}\right)\right\} \in(V)^{n}$, where $(V)^{n}$ is the $n$-th symmetric power of $V$.

The Abel map $\mathfrak{A}:(V)^{n} \rightarrow \operatorname{Jac}(V)$ puts into correspondence the divisor $\mathcal{D}$, with fixed $Z$, and the point $u \in \operatorname{Jac}(V)$, according to the rule

$$
u=\int_{\mathcal{Z}}^{x} \mathrm{~d} \mathbf{u}, \quad \text { or } \quad u_{i}=\sum_{k=1}^{n} \int_{z_{k}}^{x_{k}} \mathrm{~d} u_{i}, \quad i=1, \ldots, g .
$$

The Jacobi inversion problem is formulated as the problem of inversion of the Abel map.

The standard $\theta$ function $\theta(v \mid \tau)$ is defined by its Fourier series,

$$
\theta(v \mid \tau)=\sum_{m \in \mathbb{Z}^{g}} \exp \pi i\left\{m^{T} \tau m+2 v^{T} m\right\}
$$

The $\theta$ function possesses the following periodicity properties $\forall k \in 1, \ldots, g$ :

$$
\begin{aligned}
& \theta\left(v_{1}, \ldots, v_{k}+1, \ldots, v_{g} \mid \tau\right)=\theta(v \mid \tau), \\
& \theta\left(v_{1}+\tau_{1 k}, \ldots, v_{k}+\tau_{k k}, \ldots, v_{g}+\tau_{g k} \mid \tau\right)=\mathrm{e}^{-i \pi \tau_{k k}-2 \pi i v_{k}} \theta(v \mid \tau) .
\end{aligned}
$$

Let $w^{T}=\left(w_{1}, \ldots, w_{g}\right) \in \operatorname{Jac}(V)$ be some fixed vector. The function,

$$
\Theta(x)=\theta\left(\int_{x_{0}}^{x} \mathrm{~d} \mathbf{v}-w \mid \tau\right), \quad x \in V
$$

is called the Riemann $\theta$ function. The Riemann $\theta$ function $\Theta(x)$ is either identically 0 , or it has exactly $g$ zeros $x_{1}, \ldots, x_{g} \in V$, for which the Riemann vanishing theorem says that

$$
\sum_{k=1}^{g} \int_{x_{0}}^{x_{i}} \mathrm{~d} \mathbf{v}=w+K_{x_{0}}
$$

where $K_{x_{0}}^{T}=\left(K_{1}, \ldots, K_{g}\right)$ is the vector of Riemann constants with respect to the base point $x_{0}$. 
Definition 3.7. The fundamental Abelian $\sigma$ function is defined by the formula

$$
\sigma(z)=\frac{1}{\sqrt[4]{D(V)}} \sqrt{\frac{\pi^{g}}{\operatorname{det}(\omega)}} \exp \left\{\frac{1}{2} u^{T} \eta \omega^{-1} u\right\} \theta\left(u-K_{x_{0}} \mid \omega^{\prime} \omega^{-1}\right),
$$

$K_{x_{0}}$ is the vector of Riemann constants with the base point $x_{0}$, the function $\left.D(V)=D_{x}\left(D_{y} f(x, y)\right)\right)$ is the discriminant of the defining equation $f(x, y)=0$ of the curve $V$.

The fundamental $\sigma$ function is invariant with respect to the action of the symplectic group $\operatorname{Sp}(2 g, \mathbb{Z})$ and therefore inherits the corresponding property of the $\sigma$ function of the Weierstrass theory of elliptic functions.

Kleinian $\wp$ functions are defined as logarithmic derivatives of the fundamental $\sigma$ function

$$
\begin{gathered}
\wp_{i j}(u)=-\frac{\partial^{2} \ln \sigma(u)}{\partial u_{i} \partial u_{j}}, \wp_{i j k}(u)=-\frac{\partial^{3} \ln \sigma(u)}{\partial u_{i} \partial u_{i} \partial u_{k}} \ldots, \\
i, j, k, \ldots=1, \ldots, g .
\end{gathered}
$$

The $\wp$ functions have the following periodicity properties

$$
\wp_{\mathbf{I}}\left(u+2 \Omega\left(m, m^{\prime}\right)\right)=\wp_{\mathbf{I}}(u), \mathbf{I}=\{i, j\},\{i, j, k\}, \ldots \quad i, j, k,=1, \ldots, g,
$$

where $\Omega\left(m, m^{\prime}\right)=\omega m+\omega^{\prime} m^{\prime}$.

For the remaining results, the principal role is played by the following theorem

Theorem 3.4 (Klein). Let $\left(y\left(x_{0}\right), x_{0}\right),(y, x)$ be arbitrary distinct points on $V$ and let $\left\{\left(y_{1}, x_{1}\right), \ldots,\left(y_{g}, x_{g}\right)\right\}$ be arbitrary sets of distinct points $\in(V)^{g}$. Then the following relation is valid for every $r=1, \ldots, g$

$$
\begin{aligned}
& \sum_{i, j=1}^{g} \wp_{i j}\left(\int_{a_{0}}^{x} \mathrm{~d} \mathbf{u}-\sum_{k=1}^{g} \int_{a_{0}}^{x_{k}} \mathrm{~d} \mathbf{u}\right) \mathcal{U}_{i}(x, y) \mathcal{U}_{j}\left(x_{r}, y_{r}\right) \\
= & \frac{F\left(x, y ; x_{r}, y_{r}\right)}{\left(x-x_{r}\right)^{2}}
\end{aligned}
$$

where monomials $\mathcal{U}_{i}(x, y)$ are defined by the holomorphic differentials and $F(x, y ; z, w)$ is the polynomial entering into the definition of the fundamental 2-differential (3.9).

Proof. The proof is based on the application of the Riemann vanishing theorem to compute the integral over the boundary of the fundamental domain $\partial \Delta$

$$
\oint_{\partial \Delta} \int_{z}^{x} \mathrm{~d} \Omega(x, y ; z, w) \mathrm{d} \log \Theta(x)
$$


where $\Theta(x)$ is the Riemann $\theta$ function and $\mathrm{d} \Omega(x, y ; z, w)$ is the fundamental 2-differential.

The Kleinian formula (3.28) gives an additional theorem of a "point + divisor" kind. In the case of $g=1$ it is exactly the addition theorem for the Weierstrass $\wp$ function (function $\wp_{11}(u)$ in our notation).

Expanding the formula (3.28) at $x=1 / \xi^{n}=\infty$ we obtain from the condition of vanishing of the principal part of the poles the complete set of relations between $\wp$ functions and their derivatives. In particular, the first $n-1$ equations are polynomial in $x, y$ (written instead $x_{r}, y_{r}, r=1, \ldots, g$ ) with coefficients depending on Kleinian $\wp$ functions. Their derivatives represent an over-determined system of algebraic equations whose zeros give the solution of Jacobi inversion problem. This statement will be exemplified further by the considering of the trigonal curve.

We summarise the results of this section in the form of a proposition

Proposition 3.5. Consider a nonsingular $\left(a_{0} \equiv 1\right)$ non-degenerate $\left(d_{1} \equiv\right.$ $\left.1, \ldots, d_{n-1} \equiv 1\right)$ canonical algebraic curve of orders $(n, s)$ and genus $g$. Construct the associated Weierstrass gap sequence with g gaps. Then

- the $g$ independent canonical holomorphic differentials (3.11) are defined by monomials $\mathcal{U}_{i, j}=x^{P_{i}} y^{Q_{j}}$ whose orders $n P_{i}+s Q_{j}$ are exactly the first $g$ non-gaps in the Weierstrass gap sequence.

- The associated differentials of the second kind are given by (3.19) with the polynomials

$$
\begin{aligned}
\mathcal{R}_{i j}(x, y) & =\sum_{l=0}^{j-1} \sum_{k=\alpha_{n-l-1}}^{\alpha_{n}+1} \mathfrak{R}_{i, j, k, l} x^{k} y^{l}+y^{j} \sum_{k=i+1}^{\alpha_{n}+1} \mathfrak{R}_{i, j, k, j} x^{k} \\
& +\sum_{l=j+1}^{n-1} \sum_{k=0}^{\alpha_{n}+1} \mathfrak{R}_{i, j, k, l} x^{k} y^{l},
\end{aligned}
$$

where $\alpha_{k}=\operatorname{ord}_{x} a_{k}(x), k=0, \ldots, n, \mathfrak{R}_{i, j, k, l}=\tilde{\mathfrak{R}}_{i, j, k, l}-\tilde{\mathfrak{R}}_{k, l, i, j}$ and

$$
\tilde{\mathfrak{R}}_{i, j, k, l}=\chi\left(\begin{array}{l}
l \\
j
\end{array}\right) \sum_{p=0}^{n-1-l} \sum_{q=0}^{\alpha_{n-1-j-p}} \chi\left(\begin{array}{c}
\alpha_{n-1-l-p} \\
i+k-q+2
\end{array}\right) C\left(\begin{array}{c}
n-l-1, l-j, p \\
i+k-q-2, q, i
\end{array}\right)
$$

and

$$
\begin{aligned}
C\left(\begin{array}{l}
i, j, k \\
l, r, s
\end{array}\right) & =(-1)^{\delta_{0, i} 2^{-\delta_{0, j}} a_{i-k, l} a_{i+k+j, r}} \\
& \times \begin{cases}(-1)^{\delta_{0, i} \delta_{0, j}(n-i-j)(r-s-1),} & \text { if } k=0 \\
(-1)^{\delta_{i, k}}[k(l+r-2 s-2)+j(r-s-1)], & \text { if } k>0\end{cases}
\end{aligned}
$$

where $\delta_{i, k}$ is the Kronecker symbol and $\chi\left(\begin{array}{l}l \\ k\end{array}\right)=1$ if $l>k$ and 0 otherwise. 
Proof. The proof of the first part of the theorem is based on the corollary 3.1.1. The formula (3.29) is derived by direct transformations of the right hand side of the condition (3.18) to the form of the right hand side.

The formula (3.29) generalizes the formula given by Abel and Weierstrass for the polynomial $\mathcal{R}_{i}$ in the case of a hyperelliptic curve (4.2). In the next sections we consider as examples hyperelliptic and trigonal curves.

\section{HyPERELLIPTIC CURVE}

The hyperelliptic curve $V$ of genus $g$, given by the formula

$$
f(x, y)=y^{2}-p(x), \quad p(x)=4 x^{2 g+1}+\sum_{i=0}^{2 g} \lambda_{i} x^{i},
$$

is the canonical hyperelliptic curve of order $(2,2 g+1)$. Suppose, that the curve is nonsingular, i.e. the discriminant $D_{y}(f(x, y)) \equiv p(x)$ has no multiple zeros.

The sets of canonical holomorphic differentials and associated second kind differentials have the form [1]

$$
\mathrm{d} u_{k}=\frac{x^{k-1} \mathrm{~d} x}{y}, \quad \mathrm{~d} r_{i}=\frac{\mathrm{d} x}{4 y} \sum_{k=i}^{2 g+1-i}(k+1-i) \lambda_{k+1+i} x^{k},
$$

where $i=1, \ldots, g$. The differential of the third kind with first order poles at the points $x_{1,2}$ is defined as

$$
\mathrm{d} \Pi_{x_{1}, x_{2}}(x)=\frac{\mathrm{d} x}{2 y}\left(\frac{y+y_{1}}{x-x_{1}}-\frac{y+y_{2}}{x-x_{2}}\right) .
$$

The 2-differential of the second kind $\mathrm{d} \Omega(x, y ; z, w)$ with the unique pole along the diagonal is given by (3.10) with the polynomial

$$
F(x, y ; z, w)=2 y w+\sum_{k=0}^{g} x^{k} z^{k}\left(2 \lambda_{2 k}+(x+z) \lambda_{2 k+1}\right) .
$$

The Abel pre-image of the point $u \in \operatorname{Jac}(V)$ is given by the set $\left\{\left(y_{1}, x_{1}\right), \ldots,\left(y_{g}, x_{g}\right)\right\} \in$ $(V)^{g}$, where $\left\{x_{1}, \ldots, x_{g}\right\}$ are the zeros of the Bolza equation $[3,1]$

$$
x^{g}-x^{g-1} \wp_{g, g}(u)-x^{g-2} \wp_{g, g-1}(u)-\ldots-\wp_{g, 1}(u)=0,
$$

and $\left\{y_{1}, \ldots, y_{g}\right\}$ are given by

$$
y_{k}=-\left.\frac{\partial \mathcal{P}(x ; u)}{\partial u_{g}}\right|_{x=x_{k}} .
$$




\section{TRIGONAL CURVE}

Consider the trigonal curve. Set $d_{0}=1$, then we have

$$
\begin{aligned}
& \psi_{0}(x, y)=1, \quad \psi_{1}(x, y)=\frac{y-a_{1}(x)}{d_{1}(x)}, \\
& \psi_{2}(x, y)=\frac{y^{2}-a_{1}(x) y-a_{2}(x)}{d_{2}(x)} .
\end{aligned}
$$

Write the equations

$$
\begin{aligned}
\psi_{1}^{2} & =\alpha_{11}^{(1)} \psi_{1}+\alpha_{11}^{(2)}+\alpha_{11}^{(3)} \psi_{3} \\
\psi_{1} \psi_{2} & =\alpha_{12}^{(1)} \psi_{1}+\alpha_{12}^{(2)}+\alpha_{12}^{(3)} \psi_{3} \\
\psi_{2}^{2} & =\alpha_{22}^{(1)} \psi_{1}+\alpha_{22}^{(2)}+\alpha_{22}^{(3)} \psi_{3}
\end{aligned}
$$

By equating powers of $y$ and eliminating $\alpha_{i, j}^{(k)}$ we arrive at the equation of the curve

$$
y^{3}-r(x) d_{1}(x) y^{2}-p(x) d_{2}(x) y-q(x) \frac{d_{2}^{2}(x)}{d_{1}(x)}=0,
$$

where $d_{2}$ is divisible by $d_{1}^{2}$. We restrict ourselves to the case of the nonsingular curve $d_{1}=d_{2}=1$ of the form

$$
\begin{aligned}
f(x, y) & =0, \quad f(x, y)=y^{3}-p(x) y-q(x), \\
p(x) & =\sum_{k=0}^{g-\left[\frac{g+1}{3}\right]} p_{k} x^{k}, \quad q(x)=x^{g+1}+\sum_{k=0}^{g-1} q_{k} x^{k},
\end{aligned}
$$

with the coefficients $p_{i}, q_{j}, \in \mathbb{C}$, the fraction $\frac{g+1}{3}$ being non-reducible, and the discriminant $D_{y}(f(x, y))=27 q^{2}(x)-4 p^{3}(x)$ is assumed to be without multiple zeros.

Further we shall distinguish two cases $s=3 K+1$ and $s=3 K+2$, which we shall denote in what follows as $(I)$ and $(I I)$ respectively. For example, the curves of lowest genera of order $(3,4)$ and $(3,5)$ correspondingly yield the following Weierstrass gap sequences, in which we over-line the non-gap numbers

$$
\begin{aligned}
& \overline{0} 1,2, \overline{3,4}, 5, \overline{6,7, \ldots} \quad \text { case }(3,4), \\
& \overline{0} 1,2, \overline{3}, 4, \overline{5,6}, 7, \overline{8,9, \ldots} \quad \text { case }(3,5) .
\end{aligned}
$$

The holomorphic differentials are given by the formula (3.11) with the monomials

$$
\mathcal{U}_{i, 0}, i=0, \ldots,\left[\frac{s_{1}}{3}\right], \quad \mathcal{U}_{i, 1}, i=0, \ldots,\left[\frac{s_{2}}{3}\right]
$$


where $s_{i}, i=1,2$ are the lowest non-gaps such that $s_{i}(\bmod 3)=i<3($ see corollary 3.1.1). The associated second kind differentials are given by the formula (3.19) with the polynomials $\mathcal{R}_{i}(x, y)$ given by (3.29). In particular,

$$
\begin{aligned}
\mathcal{R}_{g}(x, y) & = \begin{cases}-x^{2 K} & \text { for }(I) \\
-x^{K} y & \text { for }(I I)\end{cases} \\
\mathcal{R}_{g-1}(x, y) & = \begin{cases}-2 y x^{K} & \text { for }(I) \\
-2 x^{2 K+1}-p_{2 K+1} x^{K} y & \text { for }(I I)\end{cases}
\end{aligned}
$$

To define the fundamental 2-differential we introduce the following polynomials

$$
\begin{aligned}
\mathcal{P}(x, z) & =\sum_{l=0}^{2 K+\varepsilon}\left(p_{2 l}+x p_{2 l+1}\right) x^{l} z^{l} \\
\mathcal{Q}(x, z) & =\sum_{l=0}^{K} q_{l}(l x+(3-l) z) z^{l-1} \\
& +z^{K+1} \sum_{l=-1}^{s-K-2} q_{K+l+2}(\beta(l) x+(3-\beta(l) z)) z^{\left[\frac{l}{2}\right]}
\end{aligned}
$$

where $\varepsilon=0$ for the case $(I)$ and $\varepsilon=1$ for the case $(I I)$ and in the summand the positive integers $\beta(l)=K-3\left[\frac{l-1}{2}\right]-1$. Note, that the polynomials, $\mathcal{P}(x, z), \mathcal{Q}(x, z)$ have the property

$$
\mathcal{P}(x, x)=p(x), \quad \mathcal{Q}(x, x)=3 q(x) .
$$

The 2-differential of the second kind $\mathrm{d} \Omega(x, y ; z, w)$ with the unique pole along the diagonal is given by (3.10) with the polynomial $F(x, y ; z, w)$ being defined by the formula

$$
\begin{aligned}
F(x, y ; z, w) & =3 y^{2} w^{2}+2 y w(\mathcal{P}(x, z)+\mathcal{P}(z, x))-w^{2} p(x)-y^{2} p(z) \\
& +w \mathcal{Q}(x, z)+y \mathcal{Q}(z, x)-\mathcal{P}(x, z) \mathcal{P}(z, x) .
\end{aligned}
$$

Denote $\pi_{i, j}(u)=\wp_{i, j}(u)-\wp_{i, j+1, j+1}(u)$. Then the solution of the Jacobi inversion problem reduces to the solution of algebraic equations

$$
\begin{aligned}
& w \sum_{i=0}^{K-1} \pi_{K+2 i+2, g} z^{i}+\sum_{i=0}^{K-1}\left(\pi_{i+1, g}+z^{g} \pi_{K+2 i+1, g}\right) z^{i}=\mathcal{R}_{g}, \\
& \sum_{i=0}^{K-1}\left(\pi_{i+1, g-1}+z^{g} \pi_{K+2 i+1, g-1}\right) z^{i}+w \sum_{i=0}^{K-1} \pi_{K+2 i+2, g-1} z^{i}=\mathcal{R}_{g-1},
\end{aligned}
$$

where $\mathcal{R}_{g}$ and $\mathcal{R}_{g-1}$ are monomials given in (5.5). Each pair of equations is reduced after elimination of $w$ to an algebraic equation of degree $g$, whose coefficients expressed in terms of the functions $\pi_{i, j}(u)$ give the solution of the Jacobi inversion problem. These equations generalizes the Bolza equation (4.5) to the case of trigonal curve. 
In particular the first and the last symmetric functions are

$$
\begin{aligned}
& s_{1}=\frac{1}{2} \wp_{g g g}-\frac{3}{2} \wp_{g-1, g} \quad \text { for }(I) \text { and }+\frac{1}{2} p_{2 K+1} \wp_{g, g} \text { for }(I I) \\
& s_{g}=\frac{\varepsilon}{2}\left[\left(\wp_{1, g} \wp_{K+2, g-1}-\wp_{1, g-1} \wp_{K+2, g}\right)+\left(\wp_{1, g, g} \wp_{K+2, g}-\wp_{1, g} \wp_{K+2, g, g}\right)\right],
\end{aligned}
$$

where in the second formula $\varepsilon=1$ for the case (I) and -1 for the case (II). In particular for the curve of order $(3,4)$ the symmetric functions are given as

$$
\begin{aligned}
& s_{1}=-\frac{3}{2} \wp_{23}+\frac{1}{2} \wp_{333} \\
& s_{2}=-\frac{1}{2} \wp_{33}\left(\wp_{22}-\wp_{233}\right)+\frac{1}{2} \wp_{23}\left(\wp_{23}-\wp_{333}\right)-\wp_{13} \\
& s_{3}=-\frac{1}{2} \wp_{33}\left(\wp_{12}-\wp_{133}\right)+\frac{1}{2} \wp_{13}\left(\wp_{23}-\wp_{333}\right)
\end{aligned}
$$

In the case of the curve of order $(3,5)$

$$
\begin{aligned}
s_{1} & =\frac{1}{2} \wp_{444}-\frac{3}{2} \wp_{34}+\frac{1}{2} p_{3} \wp_{44} \\
s_{2} & =-\frac{1}{2} \wp_{34}\left(\wp_{34}-\wp_{444}\right)+\frac{1}{2} \wp_{244}-\frac{1}{2} \wp_{23} \\
& -\frac{1}{2} \wp_{44}\left(\wp_{33}-\wp_{344}\right)+\frac{1}{2} p_{3} \wp_{24} \\
s_{3} & =-\frac{1}{2} \wp_{24}\left(\wp_{33}-\wp_{344}\right)+\frac{1}{2} \wp_{34}\left(\wp_{23}-\wp_{244}\right)-\frac{1}{2} \wp_{13} \\
& +\frac{1}{2} \wp_{144}+\frac{1}{2} p_{3} \wp_{14} \\
s_{4} & =\frac{1}{2} \wp_{34}\left(\wp_{13}-\wp_{144}\right)-\frac{1}{2} \wp_{14}\left(\wp_{33}-\wp_{344}\right)
\end{aligned}
$$

\section{DISCUSSION}

It was mentioned in the introduction that in $[4,5]$ the matrix $H=\left\{h_{i, k}\right\}_{i=1, \ldots, g+1, j=1, \ldots, g+1}$ was found for the genus $g$ hyperelliptic curve (4.1) of rank $\leq 4$ with the entries

$$
\begin{aligned}
h_{i k} & =4 \wp_{i-1, k-1}-2 \wp_{k, i-2}-2 \wp_{i, k-2} \\
& +\frac{1}{2}\left(\delta_{i k}\left(\lambda_{2 i-2}+\lambda_{2 k-2}\right)+\delta_{k, i+1} \lambda_{2 i-1}+\delta_{i, k+1} \lambda_{2 k-1}\right) .
\end{aligned}
$$

It was shown there, that the following relations are valid

$$
\begin{aligned}
& \wp_{g g i} \wp_{g g k}=-\operatorname{det} H\left[\begin{array}{l}
i, g+1, g+2 \\
k, g+1, g+2
\end{array}\right]=0, \quad i, k=1, \ldots, g, \\
& \operatorname{det} H\left[\begin{array}{l}
i, j, g+1, g+2 \\
k, l, g+1, g+2
\end{array}\right]=0, \quad i, j, k, l=1, \ldots, g,
\end{aligned}
$$




$$
\begin{aligned}
& \wp_{g g g i}=-\operatorname{det} H\left[\begin{array}{ll}
i, & g+1 \\
g+1 & g+2
\end{array}\right]+\operatorname{det} H\left[\begin{array}{l}
i, g+2 \\
g, g+2
\end{array}\right]-\operatorname{det} H\left[\begin{array}{l}
i-1, g+2 \\
g+1, g+2
\end{array}\right], \\
& i=1, \ldots, g,
\end{aligned}
$$

where $H\left[\begin{array}{l}i_{1} \\ j_{1}\end{array} \ldots{ }_{j_{n}}^{i_{m}}\right]=\left\{h_{i_{k}, j_{l}}\right\}_{k=1, \ldots, m ; l=1, \ldots, n}$ denotes an $m \times n$ sub-matrix. The first group of these relations describe the Jacobi variety $\operatorname{Jac}(V)$ as algebraic variety, the second group describes the $\operatorname{Kummer}$ variety, $\operatorname{Kum}(V)=$ $\operatorname{Jac}(V) / u \rightarrow-u$ and the third group describes the KdV hierarchy.

The techniques developed in this paper is aimed to enlarge this result to the case of arbitrary algebraic curve. We mention here only, that for the trigonal curve of order $(3,4)$ the condition of vanishing the next principal part (after those, which give the solution of Jacobi inversion problem) in the expansion of the Kleinian formula leads to the equation

$$
\wp_{3333}=6 \wp_{33}^{2}-3 \wp_{22}+4 p_{2} \wp_{33},
$$

which after double differentiation by $u_{3}$ becomes the Boussinesq equation with respect to the function $u(x, t)=2 \wp_{g, g}($ const $, t, x)$. Because the Kleinian function appears to be natural coordinates of the Boussinesq hierarchy, then the approach presented here creates insights to contribute to an algebro-geometrical description of solutions of the Boussinesq equation, $[14,9,15,18,8]$, and the trigonal generalizations of the Neumann system $[19,20,21]$.

\section{REFERENCES}

[1] H F Baker. Abel's theorem and the allied theory including the theory of theta functions. Cambridge Univ. Press, Cambridge, 1897.

[2] H F Baker. Multiply Periodic Functions. Cambridge Univ. Press, Cambridge, 1907.

[3] O Bolza. On the first and second derivatives of hyperelliptic $\sigma$ functions. Amer. Journ. Math., 17:11-36, 1895.

[4] V M Buchstaber, V Z Enolskii, and D V Leykin. Hyperelliptic Kleinian functions and applications, volume 179, pages 1-34. Advances in Math. Sciences, AMS Translations, series - 2, Moscow State University and University of Maryland, College Park, 1997.

[5] V M Buchstaber, V Z Enolskii, and D V Leykin. Kleinian functions, hyperelliptic Jacobians and applications. In S.P.Novikov and I.M.Krichever, editors, Reviews in Mathematics and Mathematical Physics, volume 10:2, pages 1-125, London, 1997. Gordon and Breach.

[6] V M Buchstaber, V Z Enolskii, and D V Leykin. Recursive family of polynomials generated by Sylvester's identity and addition theorem for hyperelliptic Kleinian functions. Funkt.Analiz.Pril, 31(4):19-32, 1997.

[7] V M Buchstaber, V Z Enolskii, and D V Leykin. Sigma functions of canonical curves. Funkt.Analiz.Pril, 33(1), 1999.

[8] R Dickson, F Gesztesy, and K Unterkoller. Algebro-geometric solutions of the Boussinesq hierarchy. Math. Nachrichten, 1998. in print.

[9] B A Dubrovin. Theta functions and nonlinear equations. Russ. Math. Surveys, 36:11$80,1981$. 
[10] V Z Enolskii, D V Leykin, and F W Nijhoff. Lattice KdV type equations and Abel theorem. 1999. In preparation.

[11] E V Ferapontov. Stationary Veselov-Novikov equation and isothermally asymptotic surfaces in projective differential geometry. 1998. Preprint.

[12] R W H T Hudson. Kummer's quartic surface. Cambridge university press, Cambridge, 1990. First published 1905.

[13] F Klein. Über hyperelliptische Sigmafunctionen. Math. Ann., 32:351-380, 1888.

[14] I M Krichever. Algebraic-geometric construction of the Zakharov-Shabat equations and their periodic solutions. Dokl.Akad.Nauk.SSR, 227:291-294, 1976.

[15] V B Matveev and A O Smirnov. Symmetric reductions of Riemann $\theta$ functions and some of their applications to the nonlinear Schrödinger equation and the Bussinesque equation. In Amer.Math.Soc.Transl.(2), volume 157, pages 227-237, 1993.

[16] F W Nijhoff and V Z Enolskii. Integrable mappings of KdV type and hyperelliptic addition theorems. In F W Nijhoff, editor, Proceedings of the $2^{\text {nd }}$ International Conference on Symmetries and Integrabilty of Difference Equations (SIDE II), pages 1-12, Canterbury, July 1996, 1997. Springer.

[17] M Noether. Zum Umkerhproblem in der Theorie der Abelschen Functionen. Math. Anal., 28:354-380, 1887.

[18] E Previato. Monodromy of Boussinesq elliptic operators. Acta Applicandae Math., 36:49-55, 1994.

[19] R J Schilling. Generalizations of the Neumann system - a curve theoretical approach part I. Comm. Pure Appl.Math., 40:455-522, 1987.

[20] R J Schilling. Generalizations of the Neumann system - a curve theoretical approach part II. Comm. Pure Appl.Math., 42:409-442, 1989.

[21] R J Schilling. Generalizations of the Neumann system - a curve theoretical approach part III. Comm. Pure Appl.Math., 45:775-820, 1992.

[22] K Weierstrass. Zur Theorie der Abelschen Functionen. Journ.reine angew.Math., 47:289-306, 1854.

[23] K Weierstrass. Gesammelte Werke, volume 4. Teübner, 1902.

Department of Mathematics, Heriot-Watt University, , Edinburgh, UK

E-mail address: J.C.Eilbeck@ma.hw.ac.uk

Theoretical Physics Division, NASU Institute of Magnetism, 36-B VerNADSKY STR., KIEV-680, 252142, UKRAINE

E-mail address: vze@imag.kiev.ua

Theoretical Physics Division, NASU Institute of Magnetism, 36-B VerNADSKY STR., KIEV-680, 252142, UKRAINE

E-mail address: dile@imag.kiev.ua 